\title{
Percepción de los estudiantes de maestro de educación infantil sobre su formación intercultural
}

Perception of Early Childhood Education Students about Their Intercultural Formation

Perception des étudiants de l'enseignant d'éducation des enfants par rapport à sa formation interculturelle

Percepção dos estudantes de formação de professores de educação infantil sobre sua formação intercultural

Fecha de recepción: 22 DE ENERO DE 2015/Fecha de aceptación: 16 DE SEPTIEMBRE DE 2016/Fecha de disponibilidad en línea: 15 DE DICIEMBRE DE 2016 Encuentre este artículo en http://magisinvestigacioneducacion.javeriana.edu.co/

\section{Resumen}

Se pretende conocer las creencias de los estudiantes de maestro de educación infantil acerca de la multiculturalidad, y comprender cómo se desarrolla la competencia intercultural en el proceso de formación inicial en la Facultad de Educación de Albacete de la Universidad de Castilla-La Mancha (UCLM, España). Los participantes fueron 172 estudiantes. El diseño se enmarca en el paradigma de Mixed Methods, definido como la integración sistemática de métodos de investigación cualitativos y cuantitativos en un estudio. A tal efecto, para realizar la investigación se elaboró un cuestionario de 25 preguntas, cuyos resultados se analizaron con el SPSS 20.0. Paralelamente, se construyeron los guiones de las entrevistas individuales y de los grupos de discusión, cuyo análisis fue efectuado con el Atlas.ti 6. La validez y fiabilidad quedaron garantizadas con técnicas como la revisión teórica, la aplicación piloto, el juicio de expertos y la aplicación del Alfa de Cronbach $(0,861)$. Los resultados mostraron que la formación de la competencia intercultural de los futuros maestros de educación infantil fue deficiente. Según los estudiantes, el plan de estudios no mejoró la competencia intercultural cognitiva, pedagógica y emotiva, pues al final de los estudios tenían prejuicios y estereotipos culturales negativos hacia las personas extranjeras, que durante el plan de estudios solo consiguieron suavizarlos, pero no eliminarlos.

\section{Palabras clave}

Actitudes y prejuicios; competencia intercultural; formación inicial; métodos mixtos de investigación; educación infantil
Escrito por Pedro Gil-Madrona Universidad de CAstilla-La Mancha Albacete, España Pedro.Gil@uclm.es

ISABEL Gómez-BARRETo Universidad de CAstilla-La Mancha Albacete, España IsabelMaria.Gomez@uclm.es

Sixto González-Víllora Universidad de CAstilla-La Mancha Cuenca, España Sixto.Gonzalez@uclm.es

\section{Transferencia a la práctica}

El aprendizaje de la competencia intercultural, entre otras, es la clave para el cambio que en estos instantes se está generando en el Espacio Europeo de Educación Superior, EEES. El dominio de esta competencia por parte del profesorado constituye uno de los elementos esenciales para afianzar la identidad europea a fin de que la integración política, socioeconómica y cultural sea un éxito. La competencia intercultural debe abordarse de manera transversal en las instituciones educativas desde la infancia. Es necesario que en la formación del profesorado se implementen contenidos y conocimientos, así como estrategias didácticas sobre cómo desarrollar estas competencias en la educación infantil. 


\section{Keywords}

Attitudes and prejudices; intercultural competence; initial teacher development; mixed research methods; early childhood education

\section{Abstract}

This research aims to know the beliefs of early childhood education student teachers about multiculturalism, and to understand how intercultural competence is developed in the process of initial teacher development in the Faculty of Education of Albacete of the University of Castilla-La Mancha (UCLM, Spain). Participants were 172 students. The design is framed in the Mixed Methods paradigm, defined as the systematic integration of qualitative and quantitative research methods in a study. For this purpose, a questionnaire of 25 questions was made, their results were analyzed by using SPSS 20.0. At the same time, the scripts of the individual interviews and of the focus group discussions were written, and they were analysed by using Atlas.ti 6 . Validity and reliability were guaranteed with techniques such as theoretical revision, pilot application, expert judgment and application of the Cronbach Alpha (0.861). Results showed that the development of the intercultural competence of future early childhood teachers was deficient. According to students, the curriculum did not improve their cognitive, pedagogic and emotional intercultural competence, because at the end of their studies they had prejudices and negative cultural stereotypes towards foreigners (these prejudices during their studies were soften, but not eliminated).

\section{Transfer to practice}

The learning of intercultural competence, among others, is the key to the change that in these moments is being generated in the European Space of Higher Education, EHEA. The mastery of this competence by teachers is an essential element to strengthen the European identity so that political, socio-economic and cultural integration could be a success. Intercultural competence must be addressed across educational institutions since childhood. Therefore, it is necessary that in the training of teachers they get to know how to educate and raise awareness about this competence in children's education.

\section{Mots clés}

Attitudes et préjugés; compétence interculturelle; formation première; méthodes mixtes de recherche; éducation des enfants

\section{Résumé}

On vise connaitre les croyances des étudiants de l'enseignant des enfants par rapport à la multiculturalité, et donc comprendre comment on développe la compétence interculturelle dans le processus de formation première à la Faculté d'Education d'Albacette de l'Université de Castille -La Manche (UCML). Les participants ont été 172 étudiants. Le dessein se fait dans le cadre du paradigme de Mixed Méthods, définit en tant que l'intégration systématique de méthodes de recherche qualitatives dans une étude. Ainsi, pour faire la recherche on a élaboré un questionnaire de 25 questions, dont les résultats ont étés analysés avec le SPSS 20.0. Parallèlement, on a fourni les desseins des entretiens individuelles et des groupes de débat, dont l'analyse été fait avec l'Atlas.ti 6. La validité et fiabilité ont été assurées avec les techniques telles que l'examen théorique, l'application pilote, le jugement d'experts et I'application de I'Alfa de Cronbach $(0,861)$. Les résultats montrent que la formation de la compétence interculturelle des futurs enseignants d'éducation des enfants a été insuffisante. Selon les étudiants, le plan d'études n'a pas amélioré la compétence interculturelle cognitive, pédagogique et émotive, car à la fin des études les étudiants avaient des préjugés et des stéréotypes culturels négatifs envers les personnes étrangers, que pendant le plan d'études seulement on a pu seulement les rendre plus souples et non pas les éliminer.
Transfert à la pratique

L'apprentissage de la compétence interculturelle, parmi d'autres, est clé pour le changement que dans ces moments à cours dans l'Espace Européen d'Education Supérieure, EEES. La maîtrise de cette compétence chez les enseignants constitue un des éléments essentiels pour consolider l'identité européen à fin de que l'intégration politique, socioéconomique et culturelle ait du succès. La compétence interculturelle doit s'aborder d'une façon transversale dans les institutions éducatives depuis l'enfance. C'est pourquoi qu'il faut que dans la formation des enseignants on implémente comment éduquer et se faire conscient par rapport à cette compétence dans l'éducation des enfants.

\section{Palavras-chave}

Atitudes e preconceitos; competência intercultural; formação inicial; métodos mistos de pesquisa; educação infantil

\section{Resumo}

Pretende-se conhecer as crenças dos estudantes de formação de professores de educação infantil a respeito da multiculturalidade, e compreender como se desenvolve a competência intercultural no processo de formação inicial na Faculdade de Educação de Albacete da Universidade de Castilla-La Mancha (UCLM, Espanha). Os participantes foram 172 estudantes. O desenho se situa no paradigma de Mixed Methods, definido como a integração sistemática de métodos de pesquisa qualitativos e quantitativos. Para realizar a pesquisa elaborou-se um questionário de 25 perguntas, cujos resultados se analisaram com o SPSS 20.0. Paralelamente, construíram-se os roteiros das entrevistas individuais e dos grupos de discussão, cuja análise foi efetuada com o Atlas.ti 6 . A validade e confiabilidade ficaram garantidas com técnicas como a revisão teórica, o aplicativo piloto, o julgamento de peritos e o aplicativo Alfa de Cronbach $(0,861)$. Os resultados mostraram que a formação da competência intercultural dos futuros professores de educação infantil foi deficiente. Segundo os estudantes, o plano de estudos não melhorou a competência intercultural cognitiva, pedagógica e emotiva, pois ao final dos estudos tinham preconceitos e estereótipos culturais negativos relacionados às pessoas estrangeiras, que durante o plano de estudos conseguiram amenizar sem eliminar.
Transferência à prática A aprendizagem da competência intercultural, entre outras, é a chave para a mudança que nestes instantes se está gerando no Espaço Europeu de Educação Superior, EEES. O domínio desta competência por parte do professorado se constitui num dos elementos essenciais para confirmar a identidade europeia a fim de que a integração política, socioeconômica e cultural seja exitosa. A competência intercultural deve ser abordada de maneira transversal nas instituições educativas desde a infância. Por isso, é necessário que na formação do professorado se implemente como educar e conscientizar sobre esta competência na educação infantil. 


\section{Introducción}

Una característica de la actual sociedad del siglo XXI es la complejidad, producto de la creciente pluralidad étnica y cultural (Sáez-Alonso, 2006). La sociedad española no escapa de este escenario social. La educación infantil tampoco está exenta de esta realidad, ya que muchos niños extranjeros conforman el escenario escolar infantil. Por consiguiente, la situación parece demandar, en buena lógica, que el profesorado de esta etapa educativa sea formado para poder proporcionar una enseñanza de calidad que tenga en cuenta la realidad multicultural y pluriétnica o multiétnica. Entendida esta realidad multiétnica como la de aquellas sociedades integradas por diferentes grupos étnicos, sin importar cultura, raza e historia, bajo una identidad social común, en el sentido de que en su seno se da la convivencia pacífica de varias culturas, etnias, lenguas, confesiones y estilos de vida.

Esta etapa educativa es fundamental para el desarrollo de los niños, al permitirles construir su personalidad, ampliar sus experiencias y favorecer su socialización, a la vez que ejerce una función compensadora de las desigualdades sociales (Gutiérrez-Cuevas, 1995, p.104). Por ende, todos los programas de formación de docentes y educadores para la educación infantil deben construir sus pilares en la investigación sobre la infancia. Ciertamente, un aspecto básico de la relevancia de la educación infantil tiene qué ver, como no podría ser de otra manera, con la formación del profesorado de esta etapa (Robalino-Campos, 2005).

En este sentido, está claro que la formación de docentes - además de desarrollar las capacidades para acompañar, desde el ámbito educativo, el proceso de vida de los niños- debe asumir la responsabilidad de alcanzar procesos tendientes a la transformación cultural y social de los contextos en los que esta transcurre. Por ello, uno de los primeros retos de la formación inicial de los docentes tiene qué ver con la creación de condiciones que hagan posibles la realidad multicultural actual (Aguado-Odina, Gil-Jaurena \& Mata-Benito, 2008).

Se habla de la formación por competencias o centrada en las competencias que los estudiantes universitarios deben alcanzar, como uno de los pilares para avanzar en el cumplimiento de los Objetivos de Bolonia de 1999 (Unión Europea, 1999) aunque, en la práctica, todavía hay escasa claridad en cuanto a la conceptualización, alcances y aplicabilidad de estas competencias. Dado que, al analizar diversos documentos, se constata la existencia de un importante vacío respecto a cuánto debe ser observable de la competencia o respecto al dónde ha de reflejarse la competencia demostrada. Algunos elementos que contribuyen a una descripción completa de la competencia como la manera de constatar el grado de desarrollo de la competencia adquirido por el estudiante y la fuente básica donde comprobar la evidencia de su logro, quedan sin definir (Irigoyen, Jiménez \& Acuña, 2011). Por ello, en el éxito del actual proyecto educativo orientado a la mejora de la enseñanza superior confluyen dos factores: el conocimiento y dominio que el profesorado tenga sobre las competencias específicas y generales del título o los títulos en que se desarrolla su ejercicio docente y el modo en que el docente sepa valorar en el estudiante los resultados que ha obtenido del proceso de aprendizaje (Cano-García, 2008). La competencia intercultural no puede estar ausente en el momento actual en la Unión Europea en la formación inicial de los docentes de educación infantil, pues no paran de llegar niños inmigrantes y refugiados a las aulas de infantil.
Descripción del artículo | Article description | Description de l'article | Artigo descrição

El artículo de investigación, derivado del proyecto "La competencia intercultural en el plan de estudios de graduado en Maestro de Educación Infantil en la Universidad de Castilla La Mancha", se enmarca en el desarrollo de las competencias en la educación superior, en concreto en la competencia intercultural, en la formación inicial de los maestros de Educación Infantil. El diseño corresponde al paradigma de Mixed Methods. Se llevó a cabo con la aplicación de un cuestionario, entrevistas y grupos de discusión en la Universidad de Castilla-La Mancha en España. 
Diversas investigaciones sobre la formación inicial de los docentes concluyen que no están siendo formados para atender tal realidad multicultural (Carrasco, Pàmies \& Bertran, 2009; Palmer-Pol, Montaño-Montaño \& Palou-Oliver, 2009; Rodríguez-Izquierdo, 2009; Vilà-Baños, 2006). Sin embargo, el éxito del desarrollo de competencia intercultural en gran medida corresponde al profesorado (Paricio-Tato, 2014).

Otras investigaciones (Latorre-Medina \& Blanco-Encomienda, 2010; Rodicio-García \& Iglesias-Cortizas, 2011) evidencian que los planes de estudios de las universidades españolas no abordan de forma explícita el desarrollo de la competencia intercultural, necesaria para atender la diversidad de los estudiantes y de sus familias. Lourdes Montero-Mesa (2001), por su parte, indica que los futuros docentes, en sus instituciones de formación, aprenden influenciados por sus concepciones previas.

En este sentido, Eugenio Hidalgo-Díez y Verónica Hidalgo-Hernández (2007) y Carlos Marcelo (2007) han puesto de manifiesto que la concepción que los futuros maestros traen a la universidad impide que la formación inicial modifique sus percepciones. Sin embargo, varios trabajos destacan la importancia atribuida a la competencia intercultural en el proceso de convergencia europea y su impacto en las reformas curriculares de los planes de estudios en la educación superior que están teniendo lugar en España (Edwards-Schachter \& López-Santiago, 2008). De ahí que el presente estudio se ocupará de conocer cómo perciben los estudiantes esta competencia en el plan de estudios que les forma como docentes de infantil.

Otros estudios sobre la formación del profesorado ante la interculturalidad (Domínguez-Garrido, 2006; Jordán, 2007; López-Reillo \& GarcíaFernández, 2006) coinciden en destacar la importancia de asegurar una instrucción para afrontar la educación intercultural. Rafaela García-López (2002, pp. 5-6) plantea que este período educativo debe ser transformado en relación con los estilos de enseñanza, la planificación de las materias, la organización de la docencia y la metodología. Pero se hace preciso conocer los estereotipos y prejuicios racistas de los profesores y de las administraciones responsables de la educación (Ortiz, 2008), ya que se está produciendo un considerable aumento de alumnado inmigrante en las aulas de la Unión Europea, como consecuencia de aquellas personas que acuden a la Unión en busca de una vida mejor o de la acogida de refugiados por las guerras en sus países de origen.

Es preciso conceptualizar la competencia intercultural. Beverly E. Cross (2005), Antonio Salvador Jiménez-Hernández (2013) y Chato Rasoal, Jakob Eklund y Eric M. Hansen (2011) coinciden en definirla como una capacidad adaptativa de integrarse eficientemente en un entorno multicultural. María Rosa Rodríguez-Izquierdo (2009) destaca que es un atributo transversal útil a todas las personas que trabajan en relación con personas de otras culturas. Requiere un conocimiento cultural, un modo de pensar, unas habilidades y unas destrezas que el educador debe poseer. A tal fin, para conseguir una competencia intercultural se tiene que producir una sinergia de los ámbitos cognitivos, emotivos y pedagógicos en el caso de los docentes.

En efecto, Guo-Ming Chen y William J. Starosta (1996) definen la competencia intercultural cognitiva y la competencia intercultural emotiva como habilidades para desarrollar un alto grado de autoconciencia y de consciencia cultural de los procesos comunicativos internos con personas de otras culturas y como la capacidad de recibir y proyectar las respuestas ante las personas de otras culturas de forma emocionalmente positiva.

Por otro lado, Eva María Aguaded-Ramírez, Pablo de la Rubia-Ruiz y Esther González-Castellón (2013) y Rebecca Spooner-Lane, Donna 
Tangen, K. Louise Mercer, Erika Hepple y Suzanne Carrington (2013) afirman que la actual formación en esta competencia debe estar provista de conocimientos pedagógicos y saberes culturales, que capacite a los docentes para enfrentarse a los contextos educativos en diversidad cultural, de ahí la competencia pedagógica intercultural.

En la etapa de educación infantil, el docente más se relaciona con la familia de sus alumnos (San Martín-Pérez, 2010; Siles-Rojas, 2003). Por ello, en nuestro estudio se ha querido conocer el valor que otorgan los estudiantes a la competencia intercultural en la formación inicial de los maestros de educación infantil. Identificar las percepciones, actitudes y creencias sobre la multiculturalidad que poseen los estudiantes de maestro en educación infantil. Y saber, desde la perspectiva de los estudiantes del programa de educación infantil, cómo se desarrolla la competencia intercultural en su vertiente cognitiva, afectiva y pedagógica en su formación inicial de la Facultad de Educación de Albacete de la Universidad de Castilla-La Mancha (UCLM, España). Esto con el fin de que, a partir de los resultados del presente estudio, cuando llegue el momento de la revisión de los planes de estudios de la formación de los maestros, se puedan acometer los cambios precisos, sin temor a caer en errores y omisiones.

\section{Método}

Contexto y participantes

Los participantes en la investigación son 172 estudiantes voluntarios (62 alumnos de primer curso, 61 de segundo curso y 49 de tercero), de la Facultad de Educación de Albacete de la Universidad de Castilla-La Mancha (UCLM, España), de un total de 290. A ellos se les aplicó un cuestionario, con una escala de valoración tipo Likert del cero al seis (0-6), donde el seis era la máxima puntuación y cero, no responde, la valoración menor. De entre los que contestaron el cuestionario, se escogieron al azar 26 alumnos que asistían regularmente a las clases y que habían superado todas las materias del curso al que pertenecían. Se descartaron, de una parte, aquellos alumnos que no asistían regularmente a clase y, de otro lado, aquellos otros que no tenían superadas todas las materias; después se eligió al azar entre aquellos alumnos que dieron su consentimiento informado (ocho de primer curso, nueve de segundo curso y nueve de tercer curso) para realizar entrevistas individuales y a 15 educandos congregados en tres grupos de cinco alumnos cada uno, para realizar tres equipos de discusión, uno por curso. Todos ellos son españoles.

\section{Procedimiento}

El diseño de investigación se enmarca en el enfoque de Mixed Methods, que se ha definido como la integración sistemática de métodos cualitativos y cuantitativos en un único caso (Maxwell \& Loomis, 2003), dado que, además de generalizar los resultados, se pretende profundizar en ellos. Se siguieron las orientaciones metodológicas de R. Burke Johnson, Anthony J. Onwuegbuzie y Lisa A. Turner (2007), James McMillan y Sally Schumacher (2005), Anthony J. Onwuegbuzie y Charles Teddlie (2003), dado que recomiendan utilizar esta estrategia metodológica en estudios de esta índole, para una mejor comprensión de un fenómeno social como el que se propone (Cook \& Reichardt, 1986).

En este sentido, todo el proceso (el diseño, la recogida de datos, el análisis de los mismos y la presentación de resultados) se ha realizado mediante la integración y la complementariedad de ambos métodos. Los resultados de los cuestionarios se han obtenido con estadísticos descriptivos 
(media, desviación típica o error típico), para ello ha sido fundamental la ayuda del programa informático SPSS 17.0. Los análisis de las entrevistas y los grupos de discusión se llevaron a cabo con la codificación, categorización y memorándum de los datos recogidos. Se han seguido las orientaciones metodológicas facilitadas por Steve J. Taylor y Robert Bogdan (1994), Renata Tesch (1990) y Robert Philip Weber (1990). A tal efecto, se han agrupado y enlazado las opiniones de los grupos de discusión (G) y de las entrevistas individuales (E). Se trianguló la información con gráficos, tablas y mapas que permitieran una mayor visualización para llegar a la integración de los datos mediante la comparación y la contrastación. Para las entrevistas y los grupos de discusión se utilizó el programa de análisis de datos cualitativos por categorización y memorándum (Atlas.ti 6). Este programa permite realizar codificaciones, recuperar textos o datos, vincular datos, efectuar búsquedas y recuentos, representar y sistematizar los datos, elaborar y contrastar teorías y conexiones entre códigos o categorías de información, elaborar clasificaciones y relacionar ideas y datos, y posibilitar la elaboración, clasificación y relación de ideas textuales. Para llevar a cabo este procedimiento se constituyó un grupo de investigación compuesto por tres personas.

En ese proceso de complementariedad metodológica, se intercalaron las opiniones vertidas en entrevistas y grupos de discusión, con los análisis cualitativos surgidos de los cuestionarios. Los juicios se efectuaron de forma intuitiva durante las fases de la investigación. Los datos obtenidos fueron tratados de la siguiente forma: cada uno de los investigadores analizó todas las entrevistas y grupos de discusión y junto a los datos cuantitativos realizó su informe de investigación. A continuación, en una reunión se procedió a poner en común los significados y los informes particulares. A partir de ahí, se llevó a cabo de nuevo la complementariedad metodológica y en un análisis en cascada se llegó al informe que aquí se presenta.

\section{Instrumentos}

Para realizar la presente investigación se elaboró un cuestionario de 25 preguntas. Estas preguntas surgieron del cuestionario de Juan Carlos Pastor-Vicedo (2010), que versó sobre la formación inicial de los maestros en Educación Física (con un Alfa de Cronbach de 0,88 ). Inicialmente, se sometió a una revisión teórica de contenido, de validez y de fiabilidad por jueces expertos. El instrumento elaborado al aplicarle el Alfa de Cronbach obtuvo un coeficiente global de 0,86.

Los ítems se contestan usando la escala de tipo Likert con valoraciones del 0-6 (6. Mucho, muchas, 5. Bastante, 4. Bien, 3. Suficiente, aceptable, 2. Poco, 1. Nada, 0. No responde). Paralelamente, se construyeron los guiones de las entrevistas individuales y de los grupos de discusión con las mismas dimensiones y preguntas formuladas en el cuestionario, para que el estudio tuviera un claro hilo conductor, tanto en los fines como en la categorización de los análisis, con el propósito de buscar la conexión, mediante procesos de triangulación, con la información vertida en los cuestionarios sobre la formación inicial de los futuros docentes en educación infantil y la formación de la competencia intercultural en el plan de estudios de su formación inicial. A tal efecto, las dimensiones de las entrevistas fueron las mismas que las empleadas en el cuestionario, es decir, en qué medida el plan de estudios desde su diseño contempla el desarrollo de la competencia intercultural en los estudiantes, conocer los prejuicios y estereotipos de los estudiantes si los hubiera, así como el desarrollo de la competencia intercultural cognitiva, emotiva y pedagógica. Así, las 25 interrogaciones que se enumeran en el cuestionario son la base de la categorización pues determinan las categorías de análisis que dan sentido a la información surgida en las entrevistas y grupos de discusión. Por tanto, se ha llevado a cabo un proceso de codificación axial, alrededor de los ejes, propiedades y dimensiones de las preguntas formuladas en el cuestionario. En los pasos del análisis se ha procedido a la identificación de relaciones (la dimensión o código A confirma, ratifica o contradice la dimensión B) entre las dimensiones creadas.

\section{Resultados}

Los resultados se presentan bajo el prisma de complementariedad metodológica y de forma global. Para ello se exponen los datos numéricos, producto del análisis de los cuestionarios, complementados con referencias textuales de entrevistas y grupos de discusión. En el texto, entre paréntesis, está expuesto el inicio del número de línea de la respuesta en entrevistas e inicio del número de línea de las respuestas en grupos de discusión trabajados en dos documentos (uno para entrevistas y dos para grupos de discusión) en el Atlas.ti.

Los resultados se presentan siguiendo las cinco dimensiones objeto de estudio. La formación de la competencia intercultural en los planes de estudios, prejuicios y estereotipos, competencia intercultural cognitiva, competencia intercultural emotiva y la pedagógica.

Los resultados son producto del análisis de los datos obtenidos del cuestionario (tabla 1), de las entrevistas individuales y los grupos de discusión. Para ello se integraron los resultados del cuestionario, de las entrevistas y de los grupos de discusión en cada una de las preguntas formuladas. 
Dimensiones del cuestionario y diagnostico por cursos

Con la intención de profundizar en el análisis descriptivo se presentan los resultados y la influencia que puede tener la variable independiente curso, dentro de cada una de las dimensiones en las cuales se estructura el cuestionario (tabla 1).

Tabla 1

Análisis de las dimensiones y los cursos estudiados

\begin{tabular}{|c|c|c|c|c|c|}
\hline \multicolumn{6}{|c|}{ Descriptivos por curso y dimensiones } \\
\hline & & $\mathbf{n}$ & Media & Desviación típica & Error típico \\
\hline \multirow[t]{4}{*}{ Dimensión 1} & $1^{\circ}$ Infantil & 62 & 3,2298 & 0,75653 & 0,09608 \\
\hline & $2^{\circ}$ Infantil & 61 & 2,7582 & 0,78789 & 0,10088 \\
\hline & $3^{\circ}$ Infantil & 49 & 2,2602 & 0,72161 & 0,10309 \\
\hline & Total & 172 & 2,7863 & 0,84798 & 0,06466 \\
\hline \multirow[t]{4}{*}{ Dimensión 2} & $1^{\circ}$ Infantil & 62 & 2,7634 & 0,63271 & 0,08035 \\
\hline & $2^{\circ}$ Infantil & 61 & 2,4891 & 0,59851 & 0,07663 \\
\hline & $3^{\circ}$ Infantil & 49 & 2,6293 & 0,58656 & 0,08379 \\
\hline & Total & 172 & 2,6279 & 0,61531 & 0,04692 \\
\hline \multirow[t]{4}{*}{ Dimensión 3} & $1^{\circ}$ Infantil & 62 & 2,8203 & 0,60991 & 0,07746 \\
\hline & $2^{\circ}$ Infantil & 61 & 2,8056 & 0,61613 & 0,07889 \\
\hline & $3^{\circ}$ Infantil & 49 & 2,9359 & 0,66756 & 0,09537 \\
\hline & Total & 172 & 2,848 & 0,62779 & 0,04787 \\
\hline \multirow[t]{4}{*}{ Dimensión 4} & $1^{\circ}$ Infantil & 62 & 3,086 & 0,63285 & 0,08037 \\
\hline & $2^{\circ}$ Infantil & 61 & 2,9945 & 0,8913 & 0,11412 \\
\hline & $3^{\circ}$ Infantil & 49 & 2,7007 & 0,68125 & 0,09732 \\
\hline & Total & 172 & 2,9438 & 0,75965 & 0,05792 \\
\hline \multirow[t]{4}{*}{ Dimensión 5} & $1^{\circ}$ Infantil & 62 & 2,6968 & 0,87571 & 0,11122 \\
\hline & $2^{\circ}$ Infantil & 61 & 2,282 & 0,72423 & 0,09273 \\
\hline & $3^{\circ}$ Infantil & 49 & 2,2857 & 0,59442 & 0,08492 \\
\hline & Total & 172 & 2,4326 & 0,77224 & 0,05888 \\
\hline
\end{tabular}

Fuente: elaboración propia

Se puede ver que la media del cuestionario de la primera dimensión es de 2,79 y está formada por las medias de primer curso $(3,23)$, de segundo curso $(2,76)$ y de tercer curso $(2,26)$. La media del cuestionario de la segunda dimensión es de 2,63, formada por las medias de los grupos de primero $(2,76)$, de segundo curso $(2,49)$ y de tercer curso $(2,63)$. La media del cuestionario de la tercera dimensión es 2,85 , formada por las medias de primero $(2,82)$, de segundo curso $(2,81)$ y de tercer curso $(2,94)$. La media del cuestionario de la cuarta dimensión es 2,94 y está constituida por las medias de los grupos de primero $(3,08)$, de segundo $(2,99)$ y de tercer curso $(2,70)$. La media del cuestionario de esta dimensión es 2,43 : el grupo de primer curso obtiene una media de 2,70, el grupo de segundo de 2,28 y el grupo de tercero de 2,29.

De esta forma, la variable curso presenta diferencias significativas en la primera dimensión $\left(\mathrm{F}_{2.78}=22.46, \mathrm{p}<0.01\right)$, en la segunda dimensión $\left(F_{2.62}=3.13, p<0.05\right)$, en la cuarta dimensión $\left(F_{2.94}=3.85, p<0.05\right)$ y en 
la quinta dimensión $\left(F_{2.43}=6.00, p<0.01\right)$. No se encuentra significatividad alguna en las diferencias de la tercera dimensión $\left(F_{2.84}=0.66, p>0.05\right)$.

Ello pone de manifiesto, en primer lugar, que a medida que los estudiantes van cursando el plan de estudios del título de grado de maestro de educación infantil perciben, cada vez más, que la formación de la competencia intercultural es escasa, porque el plan de estudios no despierta las expectativas iniciales de los estudiantes en relación con la interculturalidad. En segundo lugar, el plan de estudios consigue suavizar, en cierta forma, los estereotipos o prejuicios racistas con los que los estudiantes llegan a la universidad. Y en tercer lugar, el plan de estudios, en opinión de los estudiantes, no mejora la competencia intercultural pedagógica y emotiva de estos y los mantiene con la misma competencia intercultural cognitiva con la que entraron, por lo que tampoco el plan de estudios aporta mucho a los estudiantes en la dimensión cognitiva de la competencia. Estos resultados son análogos en los análisis de entrevistas y grupos de discusión, por lo cual, a continuación, se presentará la integración de los datos vertidos en cada una de las preguntas del cuestionario y en las entrevistas y grupos de discusión.

Integración de resultados. Primera dimensión.

La formación de la competencia intercultural en el plan de estudios

de la titulación de maestro en educación infantil

Se pretende conocer en qué medida los estudiantes de maestro de educación infantil consideran que el plan de estudios de su formación contempla la competencia intercultural. La pregunta uno del cuestionario: "¿En qué medida el plan de estudios de la titulación de maestro en educación infantil garantiza la formación para trabajar en contextos educativos interculturales?" obtiene la puntuación media más alta $(3,33)$ de esta dimensión. En las entrevistas y en los grupos de discusión aparecen ciertas discrepancias, ya que en los tres grupos de discusión y en 17 de las 26 entrevistas se apunta que el plan de estudios no posee asignaturas que formen para la competencia intercultural: En realidad, yo pienso que magisterio no nos forma para la competencia intercultural... (2:2G).

La pregunta dos: "¿Consideras que hay asignaturas que se ocupan específicamente de la educación intercultural?" obtiene la media más baja (2.64). En las entrevistas y los grupos de discusión se encuentran opiniones similares: Yo creo que ninguna, en dos o tres se hace referencia a los niños de otras culturas, pero no se profundiza. Creo que en este sentido, podemos decir que no nos han formado en torno a la interculturalidad (1:757E).

En la pregunta tres: "¿En las asignaturas del plan de estudios se plantean estrategias para promover el desarrollo de la competencia intercultural?", la media de respuesta es 2.88. Los tres grupos de discusión confirman que "no" hay tales estrategias. Ello se corrobora en las entrevistas, ya que 19 estudiantes manifiestan que hay "poca" formación en estrategias para promover el desarrollo de la competencia intercultural y cuatro respondieron que "no" hay estrategias: No, quizás algunos profesores se preocupan por señalar que encontraremos situaciones de interculturalidad, pero no está programada la formación en este aspecto (1.549 E).

La pregunta cuatro: "¿En el plan de estudios hay asignaturas específicas que trabajan métodos de investigación para estudiar la interculturalidad?" obtiene la media más baja de esta dimensión $(2,00)$. En las entrevistas y en los grupos de discusión se ratifican algunas de estas opiniones: Investigábamos en cuanto a los problemas de aprendizaje en una materia, indisciplinas entre los alumnos, pero no en cuanto a los problemas que puedan existir entre niños de diferentes culturas (1:8E). 
A la luz de los datos, y dado que el plan de estudio carece de una sublime oferta de materias y disciplinas en relación con la competencia intercultural, será preciso incorporar la competencia intercultural como competencia transversal en las reformas de los planes de estudios en los distintos módulos y materias del título.

Integración de resultados. Segunda dimensión. Prejuicios y estereotipos

Con esta dimensión se pretende identificar las percepciones, actitudes y creencias sobre la multiculturalidad que poseen los estudiantes de maestro en educación infantil.

El ítem cinco: "¿Los niños de otras culturas tienen dificultades en adquirir las normas establecidas en las clases?" obtiene una media de 2,56. Se evidencia que los estudiantes de educación infantil, objeto de estudio, piensan que los niños de otras culturas tienen "poca" dificultad para adquirir las normas en la clase. Ello se confirma en las entrevistas y en los grupos de discusión. Solo 5 estudiantes dicen que los niños extranjeros presentan dificultad para adaptarse a las normas de clase. Me parece que en la medida en que se hacen más diferentes las culturas, religión e idioma de la persona que viene es más difícil adecuarse. Creo por ejemplo que si viene una persona de Sudamérica se le hace más fácil adaptarse, ya que el idioma y la religión también influyen (1:789 E).

El ítem seis: "¿La posibilidad del éxito o fracaso escolar de los niños extranjeros está determinada por su cultura de origen?" obtiene la media $(1,95)$ más baja en esta dimensión. En las entrevistas y en los grupos de discusión se advierte que la cultura de origen no determina la posibilidad de éxito o fracaso en el rendimiento escolar: Yo creo que no tiene qué ver la cultura, más bien la familia, aunque creo que si una familia viene aquí en condiciones pésimas de vida, los padres están atentos a la escuela, ese niño no tiene por qué tener problemas en la escuela (2:131G). Sin embargo, en algunas de las respuestas de las entrevistas sí aparecen el idioma, el cambio de contexto o las condiciones de marginalidad como factores que afectan el rendimiento escolar de estos niños: Yo considero que sí, ya que según la cultura y si son familias marginales y que no se integran sí que afecta el rendimiento de los niños (1:32E).

La pregunta del cuestionario "LLas diferencias culturales de los padres de los niños extranjeros interfieren en el trabajo escolar de dichos niños?" (Ítem 7) obtiene una media de 2,71. Estas opiniones coinciden con los juicios dados en entrevistas y grupos de discusión, cuando indican que las diferencias culturales de los padres pueden influir negativa o positivamente, sobre todo dependiendo de la actitud y de la escala de valores de la cultura paterna: Sí, claro, depende de dónde venga. Porque los moros no se acostumbran y sus padres les enseñan a que no se adapten a las normas nuestras (2:215G).

El ítem ocho: "¿Las creencias que tengo de las personas de otras culturas varían según su origen de procedencia?" obtiene 2,27 de media. Este sentir se contradice con las opiniones dadas en las entrevistas y en los grupos de discusión, en las que se advierten las diferentes percepciones que poseen los estudiantes hacia las personas extranjeras, según sea su país de procedencia: Los rumanos son peligrosos (me dan miedo, porque en mi casa se metieron por el balcón a robar (1:225E); los argelinos son machistas, quieren imponer su cultura (1:566E); los colombianos dan un poco de desconfianza (2:106G); los ecuatorianos aunque son trabajadores, se dice que son poco honestos (2:187G); los chinos son muy innovadores y nos dan la sensación de invadir, eso da un poco de miedo. Son muchos (1:790E). Los gestos de los estudiantes al manifestar estas expresiones reafirman estas valoraciones y confirman la existencia de estereotipos sobre las personas pertenecientes a otras culturas, procedentes de otros países.

El ítem nueve: "¿Me agradan las personas de otras culturas?" obtiene la media más alta $(4,03)$ de esta dimensión. Sin embargo, en las entrevistas y en los grupos de discusión, de manera reiterada, hay expresiones de desagrado hacia las personas extranjeras. Se encontraron las siguientes opiniones: Imagen negativa de los argelinos (18), imagen negativa de los rumanos (23), imagen negativa de los ecuatorianos (6), imagen negativa de los colombianos (5), percepciones negativas de los chinos (5), superioridad cultural española (11). Ejemplo de ello: Somos reacios a culturas específicas como la de los árabes, por sus cuestiones o creencias religiosas y después de los atentados de Madrid se volvió más acentuada, además tienen costumbres de las que nosotros no entendemos (1:632E). Por lo tanto, aun cuando en los cuestionarios los estudiantes declaran, en su mayoría, agrado por las personas de otras culturas, en las entrevistas y en los grupos de discusiones dejan ver, con frecuencia, expresiones orales y gestuales que indican lo contrario.

La pregunta 10: "¿Se me hace más fácil confiar en un español, que en una persona de otra cultura?" obtuvo una media de 2,24. Se puede apreciar que, aun cuando el mayor porcentaje de respuesta correspondió a "poco", el resto de opiniones revela que, en diferente grado, les resulta más fácil confiar en un español que en una persona de otra cultura. En efecto, las percepciones y las emociones negativas expresadas reiterativamente por los estudiantes muestran "poca" confianza hacia los extranjeros: Los colombianos aunque son como los ecuatorianos, cercanos; dan un 
punto de desconfianza (1:109E); los ecuatorianos aunque son trabajadores, se dice que son poco honestos (2:188G), o esta otra: los chinos los hay que no se adaptan, dan problemas y generan mucha desconfianza (2:116 G). Se advierte, por tanto, que los futuros maestros de educación infantil, si bien no manifiestan opiniones racistas, sí tienen ciertas creencias en torno a un racismo sutil o encubierto. Será clave por consiguiente propiciar que hagan las prácticas de enseñanza en entornos educativos multiculturales para conocer de cerca la verdadera realidad de los niños de otras culturas.

\section{Integración de resultados. Tercera dimensión.}

La competencia intercultural cognitiva

A continuación, desde la perspectiva de los estudiantes, se explica cómo se desarrolla la competencia intercultural en sus vertientes cognitiva, afectiva y pedagógica en su formación inicial.

La percepción que tienen los estudiantes sobre el desarrollo en el plan de estudios de la competencia cognitiva intercultural, de conocimientos y habilidades que les permitan superar los posibles retos y dificultades, debido a las interacciones culturales de los niños inmigrantes. Ello se analiza con los resultados de los ítems del 11 al 17 del cuestionario. La consciencia de las propias características culturales (ítems 11 a 14) y consciencia de los procesos comunicativos (15-17).

El ítem 11: "¿Durante mi formación académica universitaria he reflexionado acerca de mi identidad cultural?" obtiene una media de 1,66. Semejante valoración aparece en las entrevistas y en los grupos de discusión: Lo poco que conocemos de estas cosas las aprende uno cuando está en la escuela, en el prácticum ante la presencia de niños extranjeros. En la universidad no nos enseñan (2:74G). Se evidencia que la práctica de la reflexión acerca de la identidad cultural de los futuros maestros de educación infantil es deficiente en su formación inicial.

La pregunta 12: "¿Los españoles poseen una superioridad cultural, con respecto a otras culturas?" obtiene la media más baja de esta dimensión $(1,65)$. Se confirma que, aunque mayoritariamente los alumnos dicen que los españoles no poseen superioridad cultural con respecto a otras culturas, al agrupar las opiniones se percibe que un grupo representativo $(33,52 \%)$ piensa que los españoles "si" poseen una superioridad cultural con respecto a otras culturas. Estas opiniones se confirman en las entrevistas y los grupos de discusión. Se aportan a continuación algunos ejemplos: Es superior a la cultura musulmana; los árabes, pero hay otras culturas superiores (1:664E). Los sudamericanos nos ven como superiores... hay quienes nos ven racistas... Así como unos nos ven superiores, como los sudamericanos, los franceses y los alemanes nos ven inferiores (2:205G). Estos testimonios guardan una estrecha relación con lo descrito en la dimensión anterior, en la que se advertía la existencia de prejuicios y estereotipos en los futuros docentes, hacia las personas de otras culturas.

El ítem 13: "¿Me siento identificado con mi cultura española?" obtuvo una media de 3,15. Si se observa con detalle la tabla, queda de manifiesto que los actuales estudiantes de educación infantil se sienten identificados con la cultura española como la religión, las costumbres, las tradiciones, los hábitos, los valores democráticos, el respeto a las libertades, los derechos humanos, la igualdad de hombres y mujeres: Yo me siento española, que soy abierta a las personas de otras culturas, porque a la edad que tengo, ya estamos acostumbrados a vivir con personas de varias culturas (1:152E). Se advierte un comportamiento sin prejuicios racistas; sin embargo, también dejan entrever, nuevamente, algunos prejuicios sutiles racistas o encubiertos ya descritos antes. 
La pregunta 14: "¿Reflexionar acerca de mi identidad cultural es una oportunidad de aprendizaje?" alcanza la mayor valoración positiva y la media más alta de esta dimensión (3.69), aun cuando en la pregunta 11 se evidencie que esta práctica es deficiente en su formación inicial como docente. Análoga opinión se encuentra en las entrevistas y grupos de discusión: Sí, considero que es una oportunidad de aprender de nosotros mismos (1:594E).

La pregunta 15: "¿Durante mi formación académica en la universidad, he reflexionado acerca de mis formas de expresión hacia personas de otras culturas?" logra una media de 3,30. En las entrevistas, 19 estudiantes dejan ver que no han tenido formación para adquirir la consciencia de sus procesos comunicativos y otro grupo (7) manifiesta haber recibido "poca" formación sobre ello. En la universidad, no; sin embargo, en mi entorno personal sí que conozco personas de otras culturas como argentinos y colombianos y me relaciono normal, pero no me he puesto a reflexionar (2:157G). Al realizar la complementariedad metodológica, se observa que los estudiantes han tenido "pocas" experiencias formativas sobre la reflexión de sus distintas maneras de expresión; sin embargo, otorgan importancia a estas destrezas.

Ante la pregunta 16: "¿Me es fácil iniciar una conversación con personas de otras culturas?", se observa, al agrupar los datos, que la mayoría de los alumnos de educación infantil tiene facilidad para iniciar la comunicación con personas de otras culturas. Al 48,80\% le resulta entre "bastante" y "suficiente" iniciar conversaciones con personas de otras culturas, 25,30\% entre "bastante" y "mucho" y el 25,90\% entre "poco" y "nada" fácil. Estos resultados coinciden con lo vertido en las entrevistas y grupos de discusión. Un grupo de estudiantes (13) refiere que le resulta fácil iniciar conversaciones con personas extranjeras: Lo encuentro bastante sencillo, me suele resultar agradable, me gusta conocer otras culturas... (1:744E). Sin embargo, tres estudiantes apuntan que tienen dificultades ocasionadas por el idioma. Creo que si tengo dificultad, con el mismo idioma sería fácil (1:742E).

La pregunta 17: "¿Considero que en la educación infantil los niños españoles perciben la diferencia cultural de sus compañeros extranjeros?" obtiene una puntuación media de 2,58. Las entrevistas y grupos de discusión coinciden con los resultados del cuestionario, ya que 20 entrevistados expresan que aun cuando perciben las diferencias de sus rasgos físicos (piel u ojos, entre otros) no los discriminan culturalmente: ...ellos no se dan cuenta si tiene otra cultura, a lo mejor se dan cuenta [de] que son más negritos, o [de] que hablan diferente, pero igual se relacionan (2:23G). Los estudiantes advierten, por tanto, que los niños de infantil no tienen conductas, procederes o actuaciones de tipo racista, discriminatorio o segregacionista con niños de otras culturas. A tal efecto, la formación inicial debe abordar la diversidad multicultural, la comunicación de los estudiantes de maestro de educación infantil con personas de otras culturas y facilitar las estrategias necesarias para mejorar las prácticas profesionales de los futuros docentes teniendo en consideración la diversidad del alumnado. En últimas, esto puede desarrollar la producción de material educativo y didáctico para el fortalecimiento de prácticas para la acción pedagógica en los primeros años de vida de todos los alumnos presentes en el aula.

Integración de resultados. Cuarta dimensión.

Competencia intercultural emotiva

En este apartado se analiza el aspecto emotivo de la competencia intercultural, como la empatía y las habilidades sociales, que son habilidades para desarrollar un alto grado de autoconciencia y de consciencia cultural 
de los procesos comunicativos, tanto internos como con personas de otras culturas, y la capacidad de recibir y proyectar las respuestas emocionales positivas antes, durante y después de las interacciones interculturales, asignándole gran valor a la comunicación intercultural. En definitiva, actitudes y conductas en las que las relaciones interpersonales, la participación, el aprendizaje permanente y el interés por el otro son elementos clave.

El ítem 18: "¿Me resultaría fácil ponerme en el lugar del padre de un niño extranjero para entender alguna situación problema en la escuela?" obtiene la media más alta $(3,45)$ de esta dimensión. Los datos cualitativos nos han permitido profundizar más en ello. Quince estudiantes consideran que tendrían dificultad a la hora de manejar un conflicto con los padres de niños extranjeros: Yo creo que es un poco difícil y más aún si es una persona que tiene otra lengua. Creo que en principio sería una posición distante, porque tú le estás imponiendo el cómo hacer las cosas de una manera como es aquí, y él igual la vería de otra manera. Habría bastante tensión (2:152G). El idioma dificulta comprender a los padres, si bien un grupo de nueve alumnos indica que tiene facilidad para comprender a los padres de los niños extranjeros. Yo creo que sí, ya que el solo hecho de pensar que vienen de otros países, desconocen las normas, o puede que desconozcan o no entienden lo que les pide el profesor, en algún momento... (1:7001E).

Durante los debates surgidos en las entrevistas y grupos de discusión se formuló la pregunta: ¿Qué emociones te produciría la interacción con los padres de los niños de otras culturas? La mayoría de estudiantes dice que esa interacción les produciría emociones positivas, como alegría, satisfacción, gozo, ternura y motivación. Por el contrario, seis estudiantes expresaron que les produciría emociones de miedo, inseguridad, ansiedad y desconfianza: Miedo: a veces, porque pienso que pueden pasar esas cosas que pasan con los gitanos. Como los rumanos que son hermanos de los gitanos, o los árabes (1:168E)... ansiedad por no saber cómo responderían ellos (2:147G). Ansiedad... Desconfianza... inseguridad... (2:223 G).

Los resultados de los cuestionarios, de las entrevistas y los grupos de discusión se complementan. Ya que, de una parte, en las entrevistas y en los grupos de discusión, en un grupo de alumnos aparecen relaciones empáticas positivas, pero, en menor medida, otro grupo de educandos expresa que estas interrelaciones les producirían desconfianza, miedo, ansiedad, al tiempo que expresa dificultad para establecer relaciones empáticas por razones de idioma y de prejuicios culturales. Ello confirma lo expuesto en los resultados de la segunda dimensión. Es decir, las percepciones y las emociones negativas expresadas reiterativamente por los estudiantes muestran "poca" confianza hacia los extranjeros. Será por tanto preciso que en la reforma del plan de estudios se incorporen contenidos de carácter emocional (empatía, reconocimiento de las emociones, habilidades sociales, motivaciones) hacia otras culturas en la formación inicial de los maestros de educación infantil, para que los futuros docentes den respuesta a los nuevos escenarios que encontrarán en las aulas.

La cuestión 19: "¿Las interacciones con niños de otras culturas en una clase de educación infantil me generan emociones distintas a las interacciones con niños españoles?" obtiene la media $(2,17)$ más baja en esta dimensión. 15 de los entrevistados reconocieron que les producía sentimientos positivos: tolerancia-interés-motivación (1:688E). Tres estudiantes manifiestan que les produciría miedo, pena y ansiedad: Generaría un poco de ansiedad para enfrentarme con las familias de los niños o miedo, por los problemas que pudieran causar y luego no saber cómo resolverlos... (1:681E). Integrando los resultados se observa que coinciden plenamente con lo expresado en la primera dimensión y en la dimensión 
de la competencia intercultural pedagógica. El profesorado que imparte docencia en las instituciones que se ocupan de formar a los futuros docentes de educación infantil tendrán que tomar buena nota de ello e incorporar contenidos didácticos en las diferentes materias para que los futuros docentes puedan abordar sus tareas académicas en la educación infantil con éxito ante la inminente multiculturalidad que se encontrarán en las aulas.

La pregunta 20: "¿Me resulta fácil el control de la clase de niños de diversas culturas?" obtiene una media de 3,2. El mayor porcentaje está entre $30,2 \%$ (suficiente) y 23,3\% (bastante): los manejaría bien, creo que entre los niños pequeños no tendría problemas, el problema son las familias (1:20E). Sin embargo, nueve estudiantes piensan que tendrían dificultad, debido a la falta de formación en el ámbito de la educación intercultural: En principio, creo que sería un poco difícil. Nos tocará aprender por nuestra cuenta, porque en realidad necesitamos saberlo, en todas las escuelas hay muchos niños extranjeros y no he aprendido a utilizar estrategias para la atención a la diversidad cultural, ya que solo nos enseñan más para niños con problemas, que con respecto a otras culturas (1:173E). Estos juicios coinciden con los que aparecen al analizar la primera dimensión.

Integración de resultados. Quinta dimensión. La competencia intercultural pedagógica

A continuación, se presentan los resultados de la competencia intercultural pedagógica.

El ítem 21: “¿Una práctica en mi formación profesional universitaria ha sido la realización de reflexiones mediante narraciones autobiográficas?" obtiene la media más baja de esta dimensión $(1,80)$. Los tres grupos de discusión coinciden en manifestar que se han realizado "muy pocas" prácticas reflexivas. En las entrevistas, 15 estudiantes responden que han obtenido "poca" formación y otros 11 refieren que "no" han tenido formación alguna: No me han pedido nunca que escriba mis reflexiones, pero sí que en algunos momentos nos comentan la importancia de reflexionar acerca de lo que hacemos, pero escribirlo no (1:705.E).

La pregunta 22: "¿Durante mi formación universitaria he aprendido a diseñar estrategias didácticas para atender la diversidad cultural?" tiene una media de 2.66. La mitad de los entrevistados expresa que "no" ha aprendido a diseñar estrategias didácticas para la atención a la diversidad cultural y la otra mitad indica que "poco": Yo creo que la universidad no nos prepara para esto (2:165 G).

Ante la pregunta 23: "¿Durante mi formación académica he tenido oportunidades de poner en práctica los conocimientos adquiridos referidos a la educación intercultural?" obtiene una media de 2,45.
Al agrupar los datos, se observa que más de la mitad de los alumnos $(63,9 \%)$ manifiesta haber tenido "poca" o "ninguna" oportunidad de poner en práctica los conocimientos adquiridos sobre educación intercultural. Estas opiniones se reafirman en las entrevistas, ya que los 26 entrevistados respondieron que han tenido "pocas" oportunidades para poner en práctica los conocimientos sobre la interculturalidad: No puedo decir que he recibido una educación intercultural y no he tenido oportunidades de poner en práctica los conocimientos teóricos sobre ello, porque lo que he recibido en sí de los profesores han sido pequeñas pinceladas, nada específico. En las prácticas de enseñanza sí he tenido alguna oportunidad de aprender sobre ello, eso sí innovando o improvisando mi actuación (1:133E). La didáctica intercultural la están construyendo desde el conocimiento práctico que han adquirido en las escuelas.

La pregunta 24: "¿Cuando diseño y desarrollo tareas prácticas, incorporo contenido y actividades de orden intercultural?" obtiene la media más alta de esta dimensión (2.88). En las entrevistas, 18 estudiantes respondieron que poseen "poca" formación para la programación de actividades interculturales en las prácticas de enseñanza y los otros siete respondieron que "no" han recibido formación al respecto. Similares opiniones aparecen en los grupos de discusión: Yo pienso que nos tenemos que buscar la vida nosotros mismos, porque hemos llegado a las escuelas infantiles en el prácticum, hemos aprendido de lo que vemos en los maestros y lo que nos interesa indagar por nuestra propia cuenta, porque a mí nunca en la universidad me dijeron cómo yo tenía que hacer una unidad didáctica que tomara en cuenta la interculturalidad (2:162G). Solo los estudiantes del tercer curso dicen que han tenido oportunidad de programar en las prácticas de enseñanza y estos también refieren que poseen "poca" o "ninguna" formación en cuanto a la programación de actividades de orden intercultural. Será preciso incorporar métodos de trabajo que se basen en las experiencias, las actividades y el juego, y se apliquen en un ambiente de afecto y confianza para potenciar su autoestima e integración social.

La cuestión 25: "¿He recibido formación en métodos de investigación para abordar situaciones problema en entornos educativos interculturales donde hay niños inmigrantes de distintos países de origen?" obtuvo 2,37 de media. Al agrupar las respuestas de "poco" y "nada" se evidencia que la mayoría de los estudiantes posee una deficiente formación en metodología de investigación para abordar situaciones problemáticas en entornos educativos interculturales. En las entrevistas y grupos de discusión, la mayoría (25) confirma que "no" ha recibido formación en ello: No nos han presentado investigaciones que traten 
sobre multiculturalidad ni de España ni de otros países $(1: 720 E)$. A tal efecto, se requiere que los planes de estudios de los maestros de educación infantil incluyan habilidades en el ámbito de la investigación, para que los futuros docentes puedan realizar investigaciones en las aulas de educación infantil que refuerzan la importancia de garantizar un clima de confianza para que los niños de distintas culturas puedan interactuar con sus pares y con las personas adultas, lo que favorecerá la estimulación del lenguaje, la coordinación motriz y el desarrollo intelectual.

\section{Discusión y conclusiones}

Alfonso Palmer-Pol, Juan José Montaño-Moreno y María Palou-Oliver (2009) detectaron una escasa presencia de competencias relacionadas con el desarrollo personal y social de los estudiantes, y hallaron que la competencia intercultural es una de las menos desarrolladas durante la formación inicial de los estudiantes universitarios en las islas Baleares. Algo que - según los resultados del presente estudio- ocurre de forma similar en la Facultad de Educación de Albacete (Castilla-La Mancha).

Ello coincide con otros estudios recientes en distintas universidades españolas (Domingo-Segovia, Gallego-Ortega, García-Aróstegui \& RodríguezFuentes, 2010; García-Aróstegui, 2008; Latorre-Medina \& Blanco-Encomienda, 2010; Rodicio-García \& IglesiasCortizas, 2011), que han puesto de manifiesto que los nuevos planes de estudios no desarrollan suficientemente las competencias profesionales de los futuros docentes en educación infantil. Silvia Carrasco, Jordi Pàmies y Marta Bertran (2009) y Rosario MéridaSerrano (2006) exponen que la mayoría de los profesionales universitarios, de las distintas ramas del conocimiento, no tiene los conocimientos psicopedagógicos necesarios para obtener la claridad que implica el modelo de formación por competencias. Esta afirmación coincide con el presente estudio, si bien aquí solo se estudia la competencia intercultural.

La opinión de los estudiantes es que no han recibido formación sobre conocimientos cognitivos, perceptivos y de comunicación frente a la diversidad cultural. Por tanto, hay ciertos prejuicios y estereotipos culturales negativos hacia las personas extranjeras. Tales conclusiones coinciden con otras investigaciones realizadas en España y Estados Unidos (Cochran-Smith, Zeichner \& Fries, 2006; Contreras, Gil-Madrona, Cecchini \& García, 2007; Fernández-Castillo \& FernándezGonzález, 2006).

En Juan Carlos Pastor-Vicedo (2010), se advierten actitudes prejuiciosas sutiles y manifiestas en los futuros docentes de educación física. Otros estudios (Kesner, 2000; Marcelo, 2007) revelan que los docentes orientan su conducta a partir del conocimiento y las creencias que tienen del inmigrante en su vida personal. Concepción Yániz (2008), al referirse a las implicaciones de incorporar diseños curriculares basados en competencia en la formación universitaria, especifica que no es posible desarrollar una competencia compleja, desprovista de un conocimiento profundo. A tal efecto, se desprende que el profesorado no tiene unos conocimientos amplios sobre la temática y no se trabaja en las aulas, de ahí que los estudiantes puedan seguir teniendo actitudes prejuiciosas sutiles y manifiestas. La formación inicial para el desarrollo de la competencia emotiva intercultural es insuficiente en todo el plan de estudios. Estos resultados son similares a los obtenidos en otras investigaciones sobre la formación inicial de los maestros en las distintas especialidades (Ramírez de la Fe \& RodríguezGonzález, 2006) y en estudiantes de psicopedagogía se observaron ausencia de emociones positivas hacia el extranjero y deficiencias en las habilidades sociales (Rodicio-García \& Iglesias-Cortizas, 2011). Rosario Mérida-Serrano (2006) ya advertía que los nuevos planes de estudios para la formación del maestro de educación infantil debían considerar un desarrollo óptimo de competencias, activar resortes emocionales, afectivos y relacionales.

La formación recibida en referencia a la competencia intercultural en la dimensión pedagógica también es escasa, si bien el prácticum favorece la construcción del conocimiento en el desarrollo de la competencia intercultural. María Purificación PérezGarcía (2008), en su trabajo sobre las competencias adquiridas por los futuros docentes desde la formación inicial, muestra igualmente que los alumnos no tienen un dominio de la competencia intercultural.

En definitiva, la competencia intercultural, en el plan de estudios que conduce a la formación de los futuros docentes de educación infantil, desde la perspectiva de los estudiantes, se está desarrollando de forma incompleta, máxime si tenemos en consideración las demandas sociales de los alumnos inmigrantes en las actuales aulas de educación infantil, ya que los futuros docentes no reciben formación en educación intercultural en asignaturas específicas ni de manera transversal, y en aquellas en las que se trabaja esta competencia no se abordan estos contenidos con la suficiente profundidad como los propios alumnos demandan. En consecuencia, los resultados de este estudio no deben ser vistos como algo negativo, pues permitirán el compromiso que deberán adquirir las universidades que asumen la formación de los profesionales y permitirán una mayor riqueza de competencias y por ende, una mayor respuesta a las demandas que la sociedad actual plantea a las escuelas infantiles. La cuestión es lograr que la interculturalidad sea 
puesta en valor y que el conjunto de personas vinculadas a la educación de los niños pequeños logre crear una sinergia positiva que incremente su eficacia, como hace ya una década proponen Margarita BartoloméPina (2004), José Luis García-Llamas (2005) o Ramón Pérez-Juste (2004). En cualquier caso, nuestro objetivo en este texto es referirnos exclusivamente al personal docente, al profesorado de educación infantil en su formación inicial y aquellos que tienen encomendadas estas tareas en las universidades.

En la idea de posibilitar el progreso en un tema de actualidad como la multiculturalidad en las escuelas infantiles y de brindar a los educadores la oportunidad de ir afinando y mejorando sus métodos, este artículo ha pugnado por analizar, con énfasis en las variables de los alumnos, futuros maestros de educación infantil en su proceso de formación inicial en relación con la formación que sobre interculturalidad están recibiendo. Un factor central ha sido la necesidad de evaluar la competencia intercultural en el plan de estudios desde la mirada de quienes están recibiendo esta formación.

Una limitación del estudio es que solamente se ha tenido en consideración la opinión de los estudiantes y en una Facultad de Educación. En el futuro, se propone hacer un estudio más amplio y recoger más información, como aumentar las Facultades de Educación en la muestra en España y en otras universidades europeas. Por otro lado, se hace preciso conocer la opinión de los profesores y administradores educativos y hacer un análisis detallado sobre los planes de estudio de los grados de maestro de la educación infantil, tanto en España como en otros países de la Unión Europea.

\section{Sobre los autores}

Pedro Gill-Madrona es maestro de educación física. Licenciado y doctor en ciencias de la educación. Premio extraordinario de doctorado. Líneas de investigación: investigación cualitativa, evaluación de programas, educación física en educación infantil, primaria y secundaria, interculturalidad, imagen y percepción corporal, obesidad y valores, emociones y habilidades sociales en educación física.

Isabel Gómez-Barreto es maestra, licenciada y doctora en educación. Líneas de investigación: interculturalidad, emociones y TIC en educación.

Sixto González-Víllora es maestro, licenciado y doctor en actividad física y deportiva. Actualmente, es decano de la Facultad de Educación de Cuenca, en la Universidad de Castilla-La Mancha. Líneas de investigación: iniciación deportiva.

\section{Referencias}

Aguaded-Ramírez, E. M.; Rubia-Ruiz, P. de la \& González-Castellón, E. (2013). La importancia de la formación del profesorado en competencias interculturales. Profesorado, Revista de Currículo y Formación del Profesorado, 17 (1), 339-365. Recuperado de http://www.ugr. es/ recfpro/rev171COL6.pdf

Aguado-Odina, M. T.; Gil-Jaurena, I. \& Mata-Benito, P. (2008). El enfoque intercultural en la formación del profesorado. Dilemas y propuestas. Revista Complutense de Educación, 19 (2), 275-292. Recuperado de http://revistas.ucm.es/index.php/RCED/article/view/ RCED0808220275A/15450

Bartolomé-Pina, M. (2004). Identidad y ciudadanía: hacia una sociedad intercultural. Bordón, Revista de Pedagogía, 56 (1), 65-79. 
Cano-García, E. (2008). La evaluación por competencias en la educación superior. Profesorado, Revista de Currículo y Formación del Profesorado, 12 (3), 1-16. Recuperado de https://www.ugr.es/ recfpro/ rev123COL1.pdf

Carrasco, S.; Pàmies, J. \& Bertran, M. (2009). Familias inmigrantes y escuelas: desencuentros estrategias y capital social. Revista Complutense de Educación, 20 (1), 55-78. Recuperado de http://revistas.ucm.es/ index.php/RCED/article/view/RCED0909120055A/15379

Chen, G.-M- \& Starosta, W. J. (1996). Intercultural Communication Competence: A Synthesis. En Brant R. Burleson \& Adrianne W. Kunkel (eds.). Communication Yearbook, 18, 353-383. London: Sage.

Cochran-Smith, M.; Zeichner, K. \& Fries, K. (2006). Estudio sobre la formación del profesorado en los Estados Unidos: descripción del informe del comité de la American Educational Research Association (AERA) sobre investigación y formación del profesorado. Revista de Educación, 340, 87-116. Recuperado de http://www.revistaeducacion.mec. es/re340/re340_04.pdf

Contreras, O. R.; Gil-Madrona, P.; Cecchini, J. A. \& García, L. M. (2007). Teoría de una educación física intercultural y realidad educativa en España. Revista Paradigma, 28 (2), 7-47. Recuperado de http:// revistas.upel.edu.ve/index.php/paradigma/article/view/1760/753

Cook, T. D. \& Reichardt, C. S. (1987). Métodos cuantitativos y cualitativos en investigación evaluativa. Madrid: Morata.

Cross, B. E. (2005). New Racism, Reformed Teacher Education, and the Same Ole' Oppression. Educational Studies: A Journal of the American Educational Studies Association, 38 (3), 263-274.

Domingo-Segovia, J.; Gallego-Ortega, J. L.; García-Aróstegui, I. \& Rodríguez-Fuentes, A. (2010). Competencias comunicativas de maestros en formación. Profesorado, Revista de Currículo y Formación del Profesorado, 14 (2), 303-323. Recuperado de http://www.ugr. es/ recfpro/rev142COL7.pdf

Domínguez-Garrido, M. C. (2006). Investigación y formación del profesorado en una sociedad intercultural. Madrid: Editorial Universitas, S.A.

Edwards-Schachter, M. \& López-Santiago, M. (2008). Competencias comunicativas e interculturales y reforma curricular en el marco de la convergencia europea. Revista Complutense de Educación, 19 (2), 369383. Recuperado de https://revistas.ucm.es/index.php/RCED/article/ viewFile/RCED0808220369A/15497

Fernández-Castillo, A. \& Fernández-González, J.-D. (2006). Valoración del prejuicio racial en la infancia: adaptación preliminar de la escala de prejuicio racial sutil y manifiesto. Infancia y Aprendizaje, 29 (3), 327342. Recuperado de http://hera.ugr.es/doi/16655199.pdf

García-Aróstegui, I. (2008). Competencias comunicativas del maestro en formación. (Tesis doctoral). Facultad de Educación, Universidad de Granada. Recuperado de http://hera.ugr.es/tesisugr/17659188.pdf

García-Llamas, J. L. (2005). Educación intercultural. Análisis y propuestas. Revista de Educación, 336, 89-109. Recuperado de http://www.revistaeducacion.mec.es/re336/re336_06.pdf

García-López, R. (2002). La diversidad cultural y la formación de los profesionales de la educación. Addenda al XXI Seminario Interuniversitario de Teoría de la Educación: Globalización, Inmigración y Educación. Universidad de Granada, noviembre de 2002. Recuperado de http:// www.redsite.es/docu/21site/a2glopez.pdf

Gutiérrez-Cuevas, P. (1995). La educación infantil: modelos de atención a la infancia. Revista Complutense de Educación, 6 (1), 102-113. Disponible 
en: http://revistas.ucm.es/index.php/RCED/article/ viewFile/RCED9595120101A/17708

Hidalgo-Díez, E. \& Hidalgo-Hernández, V. (2007). Estudio de un centro educativo multicultural de la Ciudad Autónoma de Ceuta para su mejora. Bordón, Revista de Pedagogía, 59 (4), 597610. Recuperado de https://dialnet.unirioja.es/ descarga/articulo/2582781.pdf

Irigoyen, J. J.; Jiménez, M. Y. \& Acuña, K. F. (2011). Competencias y educación superior. Revista Mexicana de Investigación Educativa, 16 (48), 243-266. Recuperado de http://www.scielo.org. mx/pdf/rmie/v16n48/v16n48a11.pdf

Jiménez-Hernández, A. S. (2013). Evaluación intercultural en la enseñanza de las lenguas extranjeras. Reid, 9, 73-95. Recuperado de http://www. ujaen.es/revista/reid/revista/n9/REID9art5.pdf

Johnson, R. Burke; Onwuegbuzie, A. J. \& Turner, L. A. (2007). Toward a Definition of Mixed Methods Research. Journal of Mixed Methods Research, 1, 112-133. Recuperado de http://www.wellsreserve. org/writable/files/ctp/Qualitative_Research/ mixed_methods.pdf

Jordán, J. A. (2007). Educar en la convivencia en contextos multiculturales. En Encarnación SorianoAyala (ed.). Educación para la convivencia intercultural, 59-94. Madrid: La Muralla.

Kesner, J. (2000). Teacher Characteristics and the Quality of Child-Teacher Relationships. Journal of School Psychology, 38 (2), 133-149. Recuperado de https://www.academia.edu/24847817/ Teacher_Characteristics_and_the_Quality_of_ Child_Teacher_Relationships

Latorre-Medina, M. J. \& Blanco-Encomienda, F. J. (2010). Competencias en la contribución de los proyectos de innovación docente a la mejora del prácticum. Revista Docencia e Investigación, 19, 79-100. Recuperado de http://www.uclm. es/varios/revistas/docenciaeinvestigacion/pdf/ numero9/LaTorre_Blanco.pdf

López-Reillo, P. \& García-Fernández, J. A. (2006). La dimensión intercultural en la educación secundaria: el modelo alternativo de formación del profesorado. En María Teresa Aguado-Odina (ed.). Actas del Congreso Internacional de Educación Intercultural: Formación del Profesorado y Práctica Escolar, 275-292. Madrid: Universidad Nacional de Educación a Distancia, UNED.

Marcelo, C. (2007). La formación docente en la sociedad del conocimiento y la información: avances y temas pendientes. Olhar de Professor, 10 (1), 63-90. Recuperado de http://www.redalyc.org/ articulo.oa?id=68410104

Maxwell, J. \& Loomis, D. (2003). Mixed Method Design: An Alternative Approach. En Abbas Tashakkori
\& Charles Teddlie (ed.). Handbook of Mixed Methods in Social and Behavioral Research, 241272. London, Thousand Oaks: Sage.

McMillan, J. \& Schumacher, S. (2005). Investigación educativa: una introducción conceptual. Madrid: Pearson/Addison Wesley.

Mérida-Serrano, R. (2006). La convergencia europea y la formación universitaria en competencias para la educación infantil. Revista de Educación, 341, 663-686. Recuperado de http://www. revistaeducacion.mec.es/re341/re341_27.pdf

Montero-Mesa, L. (2001). La construcción del conocimiento profesional docente. Rosario: Homo Sapiens Ediciones.

Onwuegbuzie, A. J. \& Teddlie, C. (2003). A Framework for Analyzing Data in Mixed Methods Research. En Abbas Tashakkori \& Charles Teddlie (ed.). Handbook of Mixed Methods in Social and Behavioral Research, 351-383. London, Thousand Oaks: Sage.

Ortiz-Cobo, M. (2008). Inmigración en las aulas: percepciones prejuiciosas de los docentes. Papers, 87, 253-268. Recuperado de https://ddd.uab. cat/pub/papers/02102862n87/02102862n8 7p253.pdf

Palmer-Pol, A.; Montaño-Moreno, J. \& Palou-Oliver, M. (2009). Las competencias genéricas en la Educación Superior. Psicothema, 21, 3, 433-438. Recuperado de http://www.psicothema.com/ pdf/3650.pdf

Palomero-Pescador, J. E. (2006). Formación inicial de los profesionales de la educación en pedagogía intercultural: una asignatura pendiente. El caso de Aragón. Revista Interuniversitaria de Formación del Profesorado, 20 (1), 207-230. Recuperado de https://dialnet.unirioja.es/descarga/ articulo/2234449.pdf

Paricio-Tato, M. S. (2014). Competencia intercultural en la enseñanza de las lenguas extranjeras. Porta Linguarum, 21, 215-226. Recuperado de http://www.ugr.es/ portalin/articulos/PL_ numero21/14\%20\%20Silvina.pdf

Pastor-Vicedo, J. C. (2010). Actitudes y estereotipos encontrados en la formación inicial del profesor de Educación Física hacia el inmigrante. (Tesis doctoral). Facultad de Educación de Toledo, Universidad de Castilla-La Mancha.

Pérez-García, M. P. (2008). Competencias adquiridas por los futuros docentes desde la formación inicial. Revista de Educación, 347, 343-367. Recuperado de http://www.revistaeducacion.mec.es/ re347/re347_16.pdf

Pérez-Juste, R. (2004). La educación en contextos multiculturales: diversidad e identidad. Bordón, Revista de Pedagogía, 56, 1, 7-23. 
Ramírez de la Fe, M. C. \& Rodríguez-González, Á. (2006). Variables predictoras de la actitud hacia los inmigrantes en la Región de Murcia (España). Anales de Psicología, 22 (1), 76-80. Recuperado de http://www.redalyc.org/pdf/167/16722110. pdf

Rasoal, C.; Eklund, J. \& Hansen, E. M. (2011). Toward A Conceptualization of Ethnocultural Empathy. Journal of Social, Evolutionary, and Cultural Psychology, 5 (1), 1-13. Recuperado de http:// psycnet.apa.org/journals/ebs/5/1/1.html

Robalino-Campos, M. (2005). ¿Actor o protagonista? Dilemas y responsabilidades sociales de la profesión docente. Revista PRELAC, Oficina Regional de Educación de la UNESCO para América Latina y el Caribe, OREALC/UNESCO, 1, 6-23. Recuperado de http://unesdoc.unesco.org/ images/0014/001446/144666s.pdf\#144709

Rodicio-García, M. L. \& Iglesias-Cortizas, M. (2011). La formación en competencias a través del Practicum: un estudio piloto. Revista de Educación, 354, 99124. Recuperado de http://www.revistaeducacion. mec.es/re354/re354_05.pdf

Rodríguez-Izquierdo, R. M. (2009). Imagen social de los inmigrantes de los estudiantes universitarios de magisterio. Revista Complutense de Educación, 20 (2), 255-274. Recuperado de http:// revistas.ucm.es/index.php/RCED/article/view/ RCED0909220255A/15273

Sáez-Alonso, R. (2006). La educación intercultural. Revista de Educación, 339, 859-881. Recuperado de http://www.revistaeducacion.mec.es/re339/ re339a37.pdf

San Martín-Pérez, J. A. (2010). La relación profesoralumno. Caminos para su mejora desde el AT [Antiguo Testamento]. Madrid: Editorial CCS.
Siles-Rojas, C. (2003). La colaboración de los padres en la escuela. Padres y Maestros, 279, 10-14. Recuperado de http://revistas.upcomillas.es/index. php/padresymaestros/article/view/2020/1753

Spooner-Lane, R.; Tangen, D.; Mercer, K. L.; Hepple, E. \& Carrington, S. (2013). Building Intercultural Competence One "Patch" At a Time. Education Research International, 2013, Article ID 394829. Recuperado de https://www.hindawi.com/journals/ edri/2013/394829/

Taylor, S. J. \& Bogdan, R. (1994). Introducción a los métodos cualitativos de investigación. Barcelona: Paidós.

Tesch, R. (1990). Qualitative Research: Analysis Types \& Software Tools. Bristol, Pennsylvania: Falmer Press.

Unión Europea (1999). Declaración de Bolonia, Declaración conjunta de los Ministros Europeos de Educación. Bolonia, Italia, 19 de junio de 1999. Recuperado de http://www.educacion.gob.es/ boloniaensecundaria/img/Declaracion_Bolonia. pdf

Vilà-Baños, R. (2006). La competencia comunicativa intercultural. Un estudio en el primer ciclo de la educación secundaria obligatoria. Revista de Investigación Educativa, 24 (2), 353-372. Recuperado de http://revistas.um.es/rie/article/ view/96891/93071

Weber, R. P. (1990). Basic Content Analysis (Quantitative Applications in the Social Sciences). Newbury Park, California: Sage Publications.

Yániz, C. (2008). Las competencias en el currículo universitario: implicaciones para diseñar el aprendizaje y para la formación del profesorado. Revista de Docencia Universitaria, 1, 2-13. Recuperado de https://dialnet.unirioja.es/descarga/articulo/2749775.pdf 